\title{
On single-time methods in relativistic gravity dynamics
}

\author{
Yu.A.Opanasyuk \\ National Technical University of Ukraine "Kyiv Polytechnical Institute" \\ 37 Peremohy Av., UA-252056 Kyiv, Ukraine
}

Received March 4, 1998

\begin{abstract}
A short outline of revived methods of an action-at-a-distance description of interacting particles is given. A new approach to the problem of motion in relativistic gravity is discussed. The approach makes an essential use of the predictive relativistic mechanics method with some phenomenological assumptions as to the character of relativistic "forces". The technique of the construction of the approximate solutions for the Currie-Hill equations is proposed. Some recurrent conditions for these solutions are proved to be necessary and sufficient. The generalized Poincaré-invariant equations of motion are derived in post-Newtonian approximations of the phenomenological formulation of the relativistic action-at-a-distance gravity for the closed system of $N$ structureless particles. Connections of these equations with those of the Lagrangian description are discussed.
\end{abstract}

Key words: relativistic mechanics, direct interaction, gravity

PACS: 04.25.-g, 04.80.Cc, 11.30.Cp

\section{Introduction}

It is customary to describe various interactions in the nature in terms of classical or quantum fields. Since Faraday, such a formulation has succeeded eminently in describing natural phenomena, particularly two fundamental interactions of classical physics, namely, electromagnetism and gravitation. The success has led to a belief that field prescription is the only way how to describe these interactions. But the field-theoretic description has both achievements and difficulties which have never been solved. The fact is that the particles interacting via fields are submitted to a kind of dynamics very different from the classical one. Fields do not propagate with the infinite velocity and the resulting dynamics is hereditary. By this we mean that the positions and velocities given at a fixed time are not sufficient data for prediction. For example, in order to determine the further states of a two-electron system moving by retarded potentials, we need information about its past behaviour: elimination of the fields by making use of the Lienard-Wiechert 
potentials does not eliminate the difficulty; the resulting equations of motion have a differential-difference structure and the problem still defies the solution. If one attempts to escape the difficulty by expanding these differential-difference equations in the Taylor series about some observer's present time, one obtains infinite-order differential equations - i.e. again a description involving an infinite number of degrees of freedom.

So, the basic difficulty of the field-theoretic description of an interparticle interaction in relativistic mechanics is the necessity of introducing an infinite number of degrees of freedom to describe the world lines of a finite number of particles. As a consequence of this, the simplest problem in the relativistic electrodynamics of interacting particles, namely, the two-body problem, has never been solved, with the exception of the circular-orbit solutions found by Schield, solutions to the two-body problem in the limit in which the mass of one of the particles goes to infinity, which is no longer a two-body problem (see, e.g., [1]-[5]).

Free or almost free from all of the aforementioned difficulties the action-at-adistance point of view was predominant from the time of Newton to the time of Maxwell and Einstein. Since Maxwell, the action-at-a-distance point of view has been largely ignored. Recent years have seen a revival of the action-at-a-distance description (see $[4,5]$ ).

Two distinct threads can be seen in this revival (see $[2,4,5]$ ). The first starts with Schwarzschild (see in [2]), Tetroge [6] and Fokker [7], and runs via Dirac (see in [2]) to the electrodynamics of Wheeler and Feynman [8], which itself is a special case of the later relativistic mechanics of Van Dam and Wigner [9,10]. This thread is marked by manifestly covariant, many-time theories. The forces between the particles act along light cones, or, in the case of Van Dam and Wigner, through the space-like region between the past and future oriented light cones. Equations of motion are coupled differential-difference equations. For the Wheeler-Feynman electrodynamics, at least this differential-difference structure can be viewed as a vestige of the field theory which has not been removed: it arises because of the finite time required for the electromagnetic field to propagate from one particle to another.

Another thread, to which the predictive relativistic mechanics (PRM) belongs, starts with a 1949 paper by Dirac [11]. It is marked by equations of motion which are coupled ordinary differential equations. In Dirac's instant form-which is the form which has been picked up and developed-the action-at-a-distance is then instantaneous, as in the ordinary nonrelativistic Newtonian mechanics. The theory is a single-time theory, rather than a many-time theory.

The modern form of PRM of the isolated systems of $N$ structureless pointlike particles, in the manifestly predictive formalism is based on two fundamental principles (see e.g. [12]):

(1) The principle of predictivity: the evolution of such a system is ruled as the system of ordinary second-order differential equations over $R^{3 N}$.

(2) The principle of relativity: the set of trajectories of such a differential system is invariant as regards the Poincaré group. 
These principles were considered incompatible for some time. However, in 1961 Havas and Plebanski [13] demonstrated the compatibility of principles (1) and (2). Later Currie [14] and Hill [1] found the necessary conditions which the accelerations of the particles must satisfy in order for the system to be a Poincaré-invariant. Bel [15] proved that such conditions are also sufficient. These conditions that constitute a system of first-order nonlinear partial differential equations which must be satisfied by the accelerations, are called in the literature "world line conditions" or Currie-Hill equations. We must say that the requirement of a special-relativistic invariance for a classical dynamical system encompasses two distinct notions: one is the identity of dynamical laws in all the inertial frames, and another is the manifest covariance. The independence of these two notions is seen most clearly within the canonical formalism to which one is led automatically if one has a Lagrangian starting point [16]-[23]. With a Lagrangian one has a definite parameter of evolution with respect to which one has differential equations of motion. From the Lagrangian description one can pass to the equivalent Hamiltonian description based on both a phase space and the idea of Poisson brackets.

In the study of relativistic $N$ particle systems with a direct interaction, several methods have been proposed and thoroughly elaborated by the corresponding streams of papers issued in the literature [17]. The common aim of most of these approaches is to carry out the so-called Dirac program [11], i.e. to construct a sympletic realization of the Poincaré group. There is a problem of space-time interpretation of the theory which remains unsolved to the end in the scheme of the relativistic Hamiltonian description of the directly interacting particle system $[23,24]$. This problem was originated by the well-known "non-interaction theorem" $[25,26]$ forbidding the identification (in the interaction zone) of canonical position variables $q_{a}^{i}(i=1,2,3$; the subscript $a=1, \ldots, N$ labels particles of the system) with covariant space coordinates $x_{a}^{i}$ of the particle world lines in the Minkowski space $M^{4}[1,22]$.

There are some papers $[20]-[22]$ treating the problems of establishing the relation of canonical and covariant variables in the frames of the classical (nonquantum) version of the relativistic direct interaction theory (RDIT). Most of the authors relate three-dimensional covariant coordinates to a certain geometric form of dynamics (see [27]), especially to the instant one. R.P. Gaida and V.I. Tretyak in [28] proposed the algorithm of constructing canonical variables in terms of covariant coordinates and their derivatives in any order of some small parameter (particularly, in the inverse speed of light $c^{-1}$ ). The main results of this thread of modern RDIT have been formulated by R.P. Gaida, Yu.B. Kluchkovsky and V.I. Tretyak [18]-[22], [27,28]. Other results have been reported in papers [23][25], [29] (see [22]).

In literature there are some results connecting the preceding threads (see $[4,5$, $14,23,28])$.

In this paper, the conditions needed to cast a given system of gravitationally interacted particles into the scheme of PRM (or RDIT) are studied. The purpose of this study is to discuss a new approach to the problem of motion in relativistic 
gravity, which makes an essential use of the aforementioned distinct threads of RDIT methods with some phenomenological assumptions as to the character of the relativistic "forces". Generalized Poincaré-invariant equations of motion are derived from some recurrent conditions, which are proved to be necessary and sufficient.

\section{Manifestly predictive formalism}

Such a denomination of the formalism was adopted by L. Bel [15] (PRM$3)$.There are some papers [4], [15]-[17], [30] treating the problem of the construction of PRM-3. Certainly, the basic principles of these approaches are almost the same (namely, principles (1) and (2) formulated in the Introduction to this paper), but each has peculiar features which may be significant from different points of view. The most convenient for our consideration approach was described by R.P. Gaida [4]. Most of the results presented in this paper have been already obtained [15]-[17], [30]. However, according to [4], we are going to give a slightly different presentation which, together with the one of [15], will provide a deeper insight into the way this formalism works.

Let $G_{r}$ be an $r$-parametric Lie group of point transformations of the Minkowski space $M^{4}$ with parameters $\lambda^{\alpha}[4]$ :

$$
\left.\begin{array}{c}
x^{\prime \mu}=\varphi^{\mu}(x, \lambda) \\
x=\left\{x^{\mu}\right\}=\{c t, r\}, \mu=0,1,2,3, \\
\lambda=\left\{\lambda^{\alpha}\right\}, \alpha=1, \ldots r .
\end{array}\right\}
$$

Infinitesimal transformations

$$
x^{\prime \mu}=x^{\mu}+\delta x^{\mu}=x^{\mu}+\xi_{\alpha}^{\mu}(x) \delta \lambda^{\alpha}+o(\delta \lambda)
$$

are defined by generators (tangent vector fields)

$$
\mathcal{X}=\xi_{\alpha}^{\mu}(x) \frac{\partial}{\partial x^{\mu}} ; \xi_{\alpha}^{\mu}(x)=\left.\frac{\partial \varphi^{\mu}(x, \lambda)}{\partial \lambda^{\alpha}}\right|_{\lambda=0},
$$

which are connected by commutational relations

$$
\left[\mathcal{X}_{\alpha}, \mathcal{X}_{\beta}\right]=c_{\alpha \beta}^{\gamma} \mathcal{X}_{\gamma}
$$

or

$$
\xi_{\alpha}^{\mu} \frac{\partial \xi_{\beta}^{\nu}}{\partial x^{\mu}}-\xi_{\beta}^{\mu} \frac{\partial \xi_{\alpha}^{\nu}}{\partial x^{\mu}}=c_{\alpha \beta}^{\gamma} \xi_{\gamma}^{\nu},
$$

where $c_{\alpha \beta}^{\gamma}$ are the structure constants of $G_{r}$. Thus, operators $\mathcal{X}_{\alpha}$ form the Lie algebra $A G_{r}$ of $G_{r}$.

For 10-parametric Poincaré group $\mathcal{P}$ the generators of time translations $\left(\mathcal{X}_{0}^{T}\right)$, space translations $\left(\mathcal{X}_{i}^{T}\right)$, space rotations $\left(\mathcal{X}_{j}^{T}\right)$ and Lorentz rotations $\left(\mathcal{X}_{j}^{L}\right)$ are given by

$$
\mathcal{X}_{0}^{T}=-\frac{\partial}{\partial t}, \mathcal{X}_{j}^{T}=-\frac{\partial}{\partial x^{j}}
$$




$$
\begin{gathered}
\mathcal{X}_{j}^{R}=-\varepsilon_{j k i} x^{k} \frac{\partial}{\partial x_{i}}, \\
\mathcal{X}_{j}^{L}=-x_{j} \frac{\partial}{\partial t}-t \frac{\partial}{\partial x^{j}},
\end{gathered}
$$

where $\varepsilon_{j k i}$ are the Levi-Civita symbols.

In this paper we put $c=\hbar=1$.

The commutation relations characteristic of the Poincaré Lie algebra are the following:

$$
\begin{gathered}
{\left[\mathcal{X}_{i}^{T}, \mathcal{X}_{0}^{T}\right]=0,\left[\mathcal{X}_{j}^{R}, \mathcal{X}_{0}^{T}\right]=0,\left[\mathcal{X}_{i}^{T}, \mathcal{X}_{j}^{T}\right]=0,} \\
{\left[\mathcal{X}_{i}^{R}, \mathcal{X}_{j}^{T}\right]=\varepsilon_{i j k} \mathcal{X}_{k}^{T},\left[\mathcal{X}_{i}^{R}, \mathcal{X}_{j}^{R}\right]=\varepsilon_{i j k} \mathcal{X}_{k}^{R},} \\
{\left[\mathcal{X}_{i}^{R}, \mathcal{X}_{j}^{L}\right]=\varepsilon_{i j k} \mathcal{X}_{k}^{L},} \\
{\left[\mathcal{X}_{i}^{L}, \mathcal{X}_{0}^{T}\right]=\mathcal{X}_{i}^{T},} \\
{\left[\mathcal{X}_{i}^{L}, \mathcal{X}_{j}^{T}\right]=\delta_{i j} \mathcal{X}_{0}^{T},\left[\mathcal{X}_{i}^{L}, \mathcal{X}_{j}^{L}\right]=-\varepsilon_{i j k} \mathcal{X}_{k}^{R}}
\end{gathered}
$$

The Poincaré covariance of the word lines of interacting particles is the main question which we are now going to discuss. PRM-3 aims to formulate the equations of motion for a system of $N$ interacting point particles in a way totally analogous to the Newtonian one. Any observer bound to an inertial frame will be able to predict the future of the system once positions and velocities of all the particles at a certain time are given. As it was mentioned above, there is a problem of a spacetime interpretation of the theory which remains unsolved to the end in the scheme of the relativistic Hamiltonian description of a directly interacting particle system $[23,24]$. Within the framework of our approach this problem is quite avoidable. To avoid a traditional consideration of the well-known "non-interaction theorem" $[25,26]$ forbidding the identification (in the interaction zone) of canonical position variables with covariant space coordinates we have from the beginning to restrict our analysis to the Newtonian-like RDIT. By this we mean that the "avoiding line" starts from the Poincaré-Lie algebra of vector fields of infinitesimal transformations acting in $E \equiv J^{\infty}\left(R \times E^{3 N}\right)$ [4] and leads straight to the restrictions imposed in the system of interacting particles by the Currie-Hill equations derived with some phenomenological assumptions as to the character of the relativistic "forces".

Generators of infinitesimal transformations of $E$ are given by [4]:

$$
X_{\alpha}=\omega_{\alpha} \frac{\partial}{\partial t}+\sum_{a=1}^{N} \sum_{\sigma=0}^{\infty} \xi_{\alpha a}^{(\sigma) i} \frac{\partial}{\partial \stackrel{\sigma^{i}}{x_{a}}}, \alpha=1, \ldots r .
$$

The vector fields $\omega_{\alpha}$ and $\xi_{\alpha a}^{(\sigma) i}$ define the infinitesimal transformations of the coordinates of the point from $E$

$$
t^{\prime}=t+\omega_{\alpha} \delta \lambda^{\alpha} ;{\stackrel{\sigma}{x_{a}}{ }^{\prime} i}^{\prime}\left(t^{\prime}\right)={ }_{x_{a}^{\sigma}}^{i}(t)+\xi_{\alpha a}^{(\sigma) i} \delta \lambda^{\alpha} .
$$


For the spatial translation group $(\alpha \equiv T)$, and for the spatial rotation $(\alpha \equiv R)$ group (none of them act on the variable $t$, i.e. $\omega_{\alpha}=0$ ) we obtain:

$$
X_{j}^{T}=-\sum \frac{\partial}{\partial x_{a}^{j}} ; X_{j}^{R}=-\varepsilon_{i j k} \sum \sum \stackrel{\sigma k}{x_{a}^{k}} \frac{\partial}{\partial \stackrel{\sigma}{x}_{a i}} .
$$

For the boost generators we define

$$
X_{j}^{L}=\sum_{a} \sum_{\sigma=0}^{\infty}\left[\frac{\mathrm{d}^{\sigma}}{\mathrm{d} t^{\sigma}}\left(-t \delta_{j k}+v_{a k} x_{a j}\right)\right] \frac{\partial}{\partial \stackrel{\sigma}{x}_{a k}} .
$$

And for $X_{0}^{T}$ we get

$$
X_{0}^{T}=\sum_{a=1}^{N} \sum_{\sigma=0}^{\infty}{ }^{\sigma+1^{k}}{ }_{a} \frac{\partial}{\partial{ }^{\sigma k}}=\frac{\mathrm{d}}{\mathrm{d} t}-\frac{\partial}{\partial t} .
$$

Inserting expressions (15)-(17) into (9)-(12) we shall see that the commutation relations characteristic of the Poincaré-Lie algebra are satisfied. Thus, transformations (15)-(17) provide a realization of the Poincaré group which plays the central role in the theory.

As we know, PRM-3 starts from the following premise: the equations of motion are "Newton-like", i.e. the positions $x_{a}^{i}$ and velocities $v_{b}^{j} \equiv \dot{x}_{b}^{j}$ of the particles in any inertial frame of reference are described by a second-order differential system:

$$
\ddot{x}_{a}^{i}-\mu_{a}^{i}(x, \dot{x}, t)=0 ; x=\left\{x_{b}^{i}(t)\right\} ; \dot{x}=\left\{x_{b}^{i}(t)\right\} .
$$

We shall also assume that accelerations $\mu_{a}^{i}$ depend on the masses of the particles $\left(m_{1}, \ldots, m_{N}\right)$.

The Poincaré-invariance of such an approach requires the existence of a realization of the Poincaré group on a configuration space with generators (13). This condition yields the identity

$$
\left.X_{\alpha}\left[\ddot{x}_{a}^{i}-\mu_{a}^{i}\{x, \dot{x}, t\}\right]\right|_{\mu}=0
$$

where symbol $\left.\right|_{\mu}$ means that expression (19) must be computed with respect to equations (18).

Inserting generators (15)-(17) into (19) we have for the accelerations a system of equations:

$$
\begin{gathered}
\frac{\partial \mu_{a}^{i}}{\partial t}=0 ; \sum_{b} \frac{\partial \mu_{a}^{i}}{\partial x_{b}^{j}}=0 \\
\sum_{b} \varepsilon_{j k l}\left(x_{b}^{k} \frac{\partial \mu_{a}^{i}}{\partial x_{b}^{l}}+\dot{x}_{b}^{k} \frac{\partial \mu_{a}^{i}}{\partial x_{b}^{l}}\right)=\varepsilon_{j n i} \mu_{a}^{n} ; \\
\sum_{b} \frac{\partial \mu_{a}^{i}}{\partial \dot{x}_{b}^{j}}+\frac{1}{c^{2}}\left\{\sum_{b}\left[r_{a b}^{j}\left(\dot{x}_{b}^{k} \frac{\partial \mu_{a}^{i}}{\partial x_{b}^{k}}+\mu_{b}^{k} \frac{\partial \mu_{a}^{i}}{\partial \dot{x}_{b}^{k}}\right)-\dot{x}_{b}^{k} \dot{x}_{b}^{j} \frac{\partial \mu_{a}^{i}}{\partial \dot{x}_{b}^{k}}\right]+2 \mu_{a}^{i} \dot{x}_{a}^{j}+\mu_{a}^{j} \dot{x}_{a}^{j}\right\}=0 .
\end{gathered}
$$


So we see that the relativistic invariance of the world lines is equivalent to the three conditions (see [4] and [2], [15]-[17]):

(I) the acceleration functions $\mu_{a}^{i}$ do not depend on $t$ explicitly;

(II) they are also invariant under space translations and behave like space vectors under rotations;

(III) they satisfy the so-called Currie-Hill equations (22).

Equations (22) were obtained by Currie [14] and later independently by Hill [1] as the necessary Poincaré-invariance conditions. Bel [15] proved that such conditions are also sufficient.

\section{The instant form of the relativistic action-at-a-distance equa- tions of motion}

In this section we show how an instant form of the Poincaré-invariant dynamics can be formulated in terms of the Currie-Hill approach to the study of relativistic gravity. The main difficulty of this approach is a nonlinear character of the system of the first-order partial differential equations (20)-(22), which disturbs the principle of the linear superposition for relativistic "forces" $\mu_{a}$.

Our intention is to describe a compatible with special relativity Newtonian-like dynamical system of $N$ gravitationally interacting structureless point-like particles. Such kind of a description, first proposed in [31], is based on some phenomenological assumptions as to the character of relativistic "forces" $\mu_{a}$. In order to develop the earlier proposed idea $[31,32]$, we are going to give a slightly different presentation which, together with the one of [33], will provide a deeper insight into the way how this approach works. Besides, we discuss a procedure which aims to construct the solutions of the Currie-Hill equations for a Newton-like system of structureless gravitating particles: some recurrent conditions for these solutions are proved to be necessary and sufficient.

Let us now consider a problem of the gravitional interaction of $N$ point-like structureless particles characterized by their masses $m_{a}$. We postulate that the motion of each of the particles must be described by equations (18). Compatibility with the special relativity imposes on (18) the conditions (20)-(22) constituting a system of partial quasilinear equations which are very difficult to handle. Nevertheless, one can find the solution by means of the guiding principle of the so-called "Dicke framework" (cited according to [34], p. 598)—Ockham (1495) razor: Nature likes things as simple as possible. Aiming in the future to use our phenomenological approach as the basis of a new theoretical frameworks for testing relativistic gravity (the descriptions of the most widely known such kind of frameworks [34][37] show that they are still not broad enough) we must choose the way of the construction of solutions, which is not only "as simple as possible", but rather "as simple as needed" at every stage of the continuously changing experimental situation. This way is a standard one and corresponds to each of the aforementioned conditions - the most convenient for our purpose is an approximate solution in the form of the power series. But all the difficulties are in the selection of physi- 
cally meaningful solutions. These solutions, as a rule, depend on small parameters: coupling constants or $c^{-1}$.

So we postulate that

$$
\mu_{a}^{i}=\sum_{p=0}^{\infty} \mu_{a}^{i}\{p\}
$$

where $\mu_{a}^{i}-i$-th component of relativistic "forces"; $a, b, \ldots=1,2, \ldots, N ; i, j, \ldots=$ $1,2,3 ; p$ - the order (of power of $\frac{v}{c}$ ) of approximation. Further, within the framework of our phenomenological description we must put that $\mu\{0\}$ corresponds to the classical (Newtonian) limit, $\mu\{1\}$ - to the first post-Newtonian (relativistic) approximation and so on. This scheme of constructing the solution may seem very trivial, but actually it is not. The fact is that at each stage of this construction there arises the necessity of a concretization of the functional dependence $\mu_{a}^{i}\{p\}$, which may be based on pure phenomenological considerations corresponding to the physical reality. And from this point of view the present approach fills us with confidence of the existence of a deep connection of this description with well verified physical models.

Let us show how one can construct the solutions of equations (22) in the case of the closed system of $N$ structureless point-like gravitating particles. Assuming the velocities to be small and the gravitational interactions to be weak, let us look for the solutions in the form (23). Starting from widely known virial correlations of the classical mechanics (see, for example, [37]), for our case of the closed system of $N$ gravitating point-like particles it is not difficult to obtain the next estimations for the orders of the average values of the variables and their derivatives, which characterize our systems of particles:

$$
\bar{v}^{2} \sim \frac{\alpha}{r}, \frac{\partial}{\partial x^{i}} \sim \frac{1}{r}, \frac{\partial}{\partial t} \sim \frac{\bar{v}}{\bar{r}}
$$

where $\alpha$ is a coupling constant (one must remember that we use a system of units in which $G=c=1$ ). From the basic principles of our approach and estimations (24) we conclude that for all orders of the $p$ power series (23) one should consider the form

$$
\mu_{a}^{i}\{p\}=\sum_{q=0}^{p} \begin{gathered}
2(p-q) \\
\mu_{a}^{i} \\
q+2
\end{gathered},
$$

where $\stackrel{n}{\mu}_{k}$ is a function of the $n$-th power of $v, k$-th power of $r_{a b}^{-1}=\left(x_{a b}^{i} x_{a b_{i}}\right)^{-\frac{1}{2}}$; $v=\dot{r}(t) ; x_{a b}^{i}=x_{a}^{i}-x_{b}^{i}$. In such a natural way, taking all the possible combinations of powers, which are allowed by the above adopted conventions, we get for every value of $p$ an appropriate set of free parameters of the formalism. Arbitrariness of a choice of these parameters is removed by the selection of definite physical restrictions.

As it turns out (see [31,32]), this approach to the "modelling" of a gravitational interaction under a definite choice of free parameters describes the conclusions of different theories of a gravitational interaction, including GR. It makes possible to use the present approach as a basis for some common formalism which is broad 
enough, as we suppose, for the planning of experimental testing of relativistic gravity.

Let define the next operators:

$$
\begin{gathered}
\mathcal{L}_{1}^{j}=-\sum_{b} \delta^{k j} \frac{\partial}{\partial v_{b}^{k}}, \\
\mathcal{L}_{2}^{j}=-2 v_{a}^{j}+\sum_{b} v_{b}^{k} v_{b}^{j} \frac{\partial}{\partial v_{b}^{k}}+\sum_{b} v_{b}^{k} x_{b a}^{j} \frac{\partial}{\partial x_{b}^{k}}, \\
\mathcal{L}_{3}^{j}\{p\}=\sum_{b} x_{b a}^{j} \mu_{b}^{k}\{p\} \frac{\partial}{\partial v_{b}^{k}}, \\
\mathcal{L}_{3}^{j}=\sum_{p=0}^{\infty} \mathcal{L}_{3}^{j}\{p\}=\sum_{b} x_{b a}^{j} \mu_{b}^{k} \frac{\partial}{\partial v_{b}^{k}}, \\
\mathcal{L}_{4}^{i}=-v_{a}^{i} ;
\end{gathered}
$$

then equations (22) take the form:

$$
\sum_{l=1}^{3} \mathcal{L}_{l}^{j} \mu_{a}^{i}+\mathcal{L}_{4}^{i} \mu_{a}^{j}=0
$$

This is one of a series of papers which discuss the possibilities of quite a new general framework for testing relativistic gravity. The differential geometrical meaning of the operators (26)-(30) will be analyzed in the future papers of this series which will be specially devoted to a unified geometrical approach allowing us to view different models for relativistic interacting particles from a common perspective. In this paper we restrict our consideration to proving a practically significant sentence.

The power series (23) is the approximate solution of equation (31) if, and only if, at any order of $p$ the next recurrence conditions take place:

$$
\mathcal{L}_{1}^{j} \mu_{a}^{i}\{p+1\}+\mathcal{L}_{2}^{j} \mu_{a}^{i}\{p\}+\sum_{k=0}^{p} \mathcal{L}_{3}^{j} \mu_{a}^{i}\{p-k\}+\mathcal{L}_{4}^{i} \mu_{a}^{j}\{p\}=0 .
$$

Indeed, the necessity of this statement follows from the fact that the Currie-Hill equations must be fulfilled at any order of $v$ and $r$ as it is required by decompositions (25). Taking into account (25) and putting (23) into equation (31), after calculations we get the series of recurrence conditions (32).

In order to prove condition (32) to be sufficient, one must denote the left-hand part of $(32)$ as $\mathcal{L}\{p\}$ and compute the sum $\sum_{p=0}^{\infty} \mathcal{L}\{p\}$. Not until one adds to this sum the zero terms $\mathcal{L}_{1}^{j} \mu^{i}\{0\}$, taking into account the properties of the operators (26)-(30), can one be sure that the obtained result is identical with the CurrieHill equations in the form (31). Thus, the recurrence conditions (32) are proved to be necessary and sufficient, whereby providing the required Poincaré-invariant description, they themselves are suitable restrictions on the arbitrary parameters 
which permit us to construct approximate solutions in any needed order of the post-Newtonian approximations. Such a recurrent technique, together with some phenomenological assumption as to the character of relativistic "forces" allows us almost completely to compensate lack of the manifest solutions of the Currie-Hill equations.

An important application of these results, quite suited for solar-system tests, is obtained from the relations (24), (25) and (32) under $p=0,1$, then, according to $(24)$ and $(25)$,

$$
\begin{aligned}
& \underset{2}{\mu_{a}^{i}}=\sum_{b \neq a} \frac{m_{b} x_{a b}^{i}}{r_{a b}^{3}} \\
& \stackrel{2}{\mu_{a}^{i}}=\sum_{b \neq a} \frac{m_{b}}{r_{a b}^{3}}\left\{x _ { a b } ^ { i } \left[b_{1}\left(\mathbf{v}_{a}\right)^{2}+b_{2}\left(\mathbf{v}_{b}\right)^{2}+b_{3}\left(\mathbf{v}_{a} \mathbf{v}_{b}\right)\right.\right. \\
& +b_{4} \frac{\left(\mathbf{r}_{a b} \mathbf{v}_{a}\right)^{2}}{r_{a b}^{2}}+b_{5} \frac{\left(\mathbf{r}_{a b} \mathbf{v}_{b}\right)^{2}}{r_{a b}^{2}}+b_{6} \frac{\left(\mathbf{r}_{a b} \mathbf{v}_{a}\right)\left(\mathbf{r}_{a b} \mathbf{v}_{b}\right)}{r_{a b}^{2}} \\
& \left.+v_{a}^{i}\left[c_{1}\left(\mathbf{v}_{a} \mathbf{r}_{a b}\right)+c_{2}\left(\mathbf{v}_{b} \mathbf{r}_{a b}\right)\right]+v_{b}^{i}\left[d_{1}\left(\mathbf{v}_{a} \mathbf{r}_{a b}\right)+d_{2}\left(\mathbf{v}_{b} \mathbf{r}_{a b}\right)\right]\right\}, \\
& \mu_{3}^{\mu_{a}^{i}}=\sum_{b \neq a} \frac{m_{b}}{r_{a b}^{3}} x_{a b}^{i}\left\{a_{1} \frac{m_{a}}{r_{a b}}+a_{2} \frac{m_{b}}{r_{a b}}+a_{3} \sum_{c \neq a, b} \frac{m_{c}}{r_{b c}}\right. \\
& \left.+a_{4} \sum_{c \neq a, b} \frac{m_{c}}{r_{a c}}+a_{5} \sum_{c \neq a, b} m_{c} \frac{\left(\mathbf{r}_{a b} \mathbf{r}_{b c}\right)}{r_{b c}^{3}}+a_{6} \sum_{c \neq a, b} m_{c} \frac{\left(\mathbf{r}_{b c} \mathbf{r}_{a c}\right)}{r_{a c}^{3}}\right\} \\
& +\sum_{b \neq a} \frac{m_{b}}{r_{a b}}\left\{a_{7} \sum_{c \neq a, b} \frac{m_{c} x_{b c}^{i}}{r_{b c}^{3}}+a_{8} \sum_{c \neq a, b} \frac{m_{b} x_{a c}^{i}}{r_{a b}^{2} r_{a c}}+a_{9} \sum_{c \neq a, b} \frac{m_{c} x_{b c}^{i}}{r_{a b}^{2} r_{b c}}+\right. \\
& \left.+x_{a b}^{i}\left[a_{10} \sum_{c \neq a, b} \frac{m_{c}\left(\mathbf{r}_{a b} \mathbf{r}_{a c}\right)}{r_{a b}^{4} r_{a c}}+a_{11} \sum_{c \neq a, b} \frac{m_{c}\left(\mathbf{r}_{a b} \mathbf{r}_{b c}\right)}{r_{a b}^{4} r_{b c}}\right]\right\} .
\end{aligned}
$$

The condition (32) allows us to express all the arbitrary parameters by means, for example, of

$$
b_{2}=\beta_{a b}, b_{4}=\gamma_{a b}, c_{1}=\delta_{a b},
$$

then, after calculations we obtain the approximate Poincaré-invariant equations of motion of the system of $N$ gravitationally interacting structureless point-like particles:

$$
\begin{aligned}
\ddot{x}_{a}^{i}={\stackrel{\mu}{\mu_{a}^{i}}}_{3} & +\sum_{b \neq a} \frac{m_{b}}{r_{a b}^{3}}\left\{x_{a b}^{i}\left[v_{a}^{2}-\beta_{a b} v_{a b}^{2}-\gamma_{a b} \frac{\left(\mathbf{r}_{a b} \mathbf{v}_{a b}\right)^{2}}{r_{a b}^{2}}+\frac{3}{2} \frac{\left(\mathbf{r}_{a b} \mathbf{v}_{a}\right)^{2}}{r_{a b}^{2}}\right]\right. \\
& \left.+v_{a b}^{i}\left[\delta_{a b}\left(\mathbf{v}_{a b} \mathbf{r}_{a b}\right)+\left(\mathbf{v}_{a} \mathbf{r}_{a b}\right)\right]\right\} .
\end{aligned}
$$

In the conclusion of this section it can be noted that the analogous equations of GR arise from (37) under the following values of the formalism parameters [31,32]:

$$
\beta_{a b}=2, \gamma_{a b}=0, \delta_{a b}=4 .
$$

Then, one has no trouble in the choice of the coefficient in that term (35), which is not restricted in this approximation by conditions (32). 


\section{Single-time relativistic Lagrangians in the phenomenologi- cal approach to the relativistic gravity}

The aim of this section is to formulate the results which were obtained for the single-time relativistic Lagrangian description of the gravitational interaction in the approximation of the concluding part of the previous section.

The existing theoretical frameworks (see, for example, [34]-[36]) are well suited for the analysis of high-precision solar-system tests which may prevail in the coming decade. Nevertheless, in our opinion they are still not broad enough. In the phenomenological approach to this problem [31,32] a very significant role will be played by a technique which is connected with the method of the Lagrangian mechanics, brilliantly suited for such kind of the analysis.

As it turns out, the existence for equation (37) of the post-Newtonian Lagrangian [31]

$$
\begin{array}{r}
L=\frac{1}{2} \sum_{a} m_{a}\left(v_{a}^{2}+\alpha_{a}^{\mathrm{L}} v_{a}^{4}\right)+\frac{1}{2} \sum_{a} \sum_{b \neq a} \frac{m_{a} m_{b}}{r_{a b}} \\
\times\left[1+\beta_{a b}^{\mathrm{L}} v_{a}^{2}+\gamma_{a b}^{\mathrm{L}}\left(\mathbf{v}_{a} \mathbf{v}_{b}\right)+\delta_{a b}^{\mathrm{L}} \frac{\left(\mathbf{r}_{a b} \mathbf{v}_{a}\right)^{2}}{r_{a b}^{2}}+\sigma_{a b}^{\mathrm{L}} \frac{m_{a}+m_{b}}{r_{a b}}\right] \\
+\sum_{a} \sum_{b \neq a} \sum_{c \neq a, b} \rho_{a b c}^{\mathrm{L}} \frac{m_{a} m_{b} m_{c}}{r_{a b} r_{a c}}
\end{array}
$$

where $\gamma_{[a b]}^{\mathrm{L}}=\delta_{[a b]}^{\mathrm{L}}=\sigma_{[a b]}^{\mathrm{L}}=\rho_{[a b]}^{\mathrm{L}}=0$, obeys the conditions:

$$
\begin{gathered}
\beta_{a b}-\frac{1}{2} \delta_{a b}+\frac{2}{3} \gamma_{a b}=0, \\
\beta_{[a b]}+\frac{1}{2} \delta_{[a b]}=0 .
\end{gathered}
$$

Deriving from (39) the Euler-Lagrange equations and imposing on them the requirement of the Poincaré-invariance by means of the previously described technique, we have the following conditions which are necessary and sufficient in the adopted approximation:

$$
\begin{aligned}
\alpha_{a}^{\mathrm{L}}=\frac{1}{4}, \gamma_{a b}^{\mathrm{L}}+\beta_{\{a b\}}^{\mathrm{L}} & =-\frac{1}{2}, \varepsilon_{a b}^{\mathrm{L}}+\delta_{\{a b\}}^{\mathrm{L}}=-\frac{1}{2}, \\
\delta_{\{a b\}}^{\mathrm{L}} & =-\beta_{\{a b\}}^{\mathrm{L}} .
\end{aligned}
$$

Conditions (42) and (43) conform to the results of papers [19]-[21] and [38], in which relativistic Lagrangians are derived, respectively, from the condition of quasiinvariancy of the Lagrangian and from the manifestly invariant Fokker-type action. We must emphasize that the existence of a Lagrangian (39) in the case of the Poincaré-invariant description is conditioned by an approximate character of the problem [20,38].

In our present investigation of the single-time Lagrangian description, as in the previous one [31], we are widely guided by the fundamental results of papers [4], [18]-[28], [38,39]. 


\section{Conclusions}

Free or almost free from some of the basic difficulties of the field-theoretical description (see Introduction to this paper) the action-at-a-distance point of view has seen a revival in the recent years. One of the threads of this revival, namely, dealing with a single-time formalism, is in our opinion quite suited for the analysis of high-precision solar-system tests which may prevail in the coming decade and for the analysis of the frameworks for testing the relativistic gravity. The importance of this analysis is clear: although there are many new experimental possibilities, the cost of carrying each one out in terms of manpower and money is very high. For this reason it is crucial that we have as good a theoretical framework as possible for comparing the relative values of various experiments - and for proposing new ones which might have been overlooked [34]-[36].

In this paper we continue the earlier proposed [31]-[33] phenomenological description of relativistic gravity. The main attention is paid to single-time methods of investigations. The reasons for this are evident: an introduction of a unique time parameter allows us to bring the theory based on the field-theoretical description closer to the form of the classical non-relativistic mechanics and to establish its relationship with other approaches to the theory of direct interactions (see $[4,38]$ ).

The generalized Poincaré-invariant approximate equations, as we believe, are very convenient in the analysis of the basic gravidynamical experiments in the Solar system. The knowledge of the Lagrangian makes it possible to obtain a complete description of a system (at least classical) by means of more or less standard methods. In the present paper we study the connections of the derived equations with those of the Lagrangian description.

Further, one may hope to obtain a deeper connection between the distinct threads of RDIT and to view different models for relativistic interacting particles from a common perspective.

\section{Acknowledgments}

I am grateful to Academician I.R. Yukhnovskii for the invitation to take part in this issue of the journal dedicated to Professor R.P. Gaida. I wish to thank V.I. Zhdanov and K. Pyragas for their support of the whole of my work. I am indebted to A.N. Alexandrov, Yu.N. Kudrya, I.Yu. Izotova, and S.L. Parnovskii for their steady interest in these investigations. It is a pleasure to acknowledge many stimulating discussions with R.P. Gaida, Yu.B. Kluchkovsky, and V.I. Tretyak.

\section{References}

1. Hill R.M. Instantaneous action-at-a-distance in classical relativistic mechanics // J. Math. Phys., 1967, vol. 8, No 2, p. 201-220.

2. Hill R.M. The origins of predictive relativistic mechanics // Lect. Notes Phys., 1982, vol. 162 , p. 104-134. 
3. Droz-Vincent Ph. Relativistic systems of interacting particles // Phys. Scr., vol. 2, No 4-5, p. 129-134.

4. Gaida R.P. Quasirelativistic systems of the interacting particles // Phys. EChAYa, 1982, vol. 13, No 2, p. 427-493 (in Russian).

5. Vladimirov Yu.S., Turygin A.Yu. Theory of Direct Interparticle Interaction. Moscow, Energoizdat, 1986 (in Russian).

6. Tetrode H. Über den Wirkungzusammenhang der Welt. Eine Erweiterung der klassischen Dymanik // Zs. Phys., 1922, vol. 10, p. 317-328.

7. Fokker A.D. Ein Invarianter Variationsatz fur die Bewegung Mehrerer Elektrischer Massenteuchen // Zs. Phys., 1929, vol. 28, No 5-6, p. 388-393.

8. Wheeler J.A., Feynman R.D. Classical electrodynamics in terms of direct interparticle action // Rev. Mod. Phys., 1949, vol. 21, No 3, p. 425-433.

9. Van Dam H., Wigner E.D. Classical relativistic mechanics of interacting particles // Phys. Rev., 1965, vol. 138, No 6, p. 1576-1582.

10. Van Dam H., Wigner E.D. Instantaneous and asymptotic conservation laws for classical relativistic mechanics of interacting point particles // Phys. Rev., 1966, vol. 142, No 4, p. 838-843.

11. Dirac P.A.M. Forms of relativistic dynamics // Rev. Mod. Phys., 1949, vol. 21, No 3, p. $392-399$.

12. Martin J., Sanz J.L. Slow motion approximation in predictive relativistic mechanics // J. Math. Phys., 1978, vol. 19, No 9, p. 1881-1891.

13. Havas P., Plebansky J. Relativistic dynamics and Newtonian causality // Bull. Amer. Phys. Soc., 1961, vol. 5, No 6, p. 433.

14. Currie D.G. Poincaré-invariant equations of motion for classical particles // Phys. Rev., 1966, vol. 142, No 4, p. 817-824.

15. Bel L. Dynamique des systemes de N particules ponctuelles on relativite restreinte // Ann. Inst. H. Poincaré, 1970, vol. A12, No 3, p. 307-321.

16. Sudarshan E.C.G., Mukunda N., Goldberg J.N. Constraint dynamics of particles world lines // Phys. Rev. D, 1981, vol. 23, No 10, p. 2218-2230.

17. Iranzo V., Llosa J., Molina A., Marques F. Comparison of several approaches to the relativistic dynamics of directly interacting particles // Ann. Phys. (N.Y.), 1983, vol. 150, No 1, p. 114-149.

18. Gaida R.P. On the Hamiltonian formulation of the instantaneous action-at-a-distance theory in relativistic classical two-body problem./Preprint of the Institute of Theoretical Physics, ITP-74-145E, Kiev 1974, 18 p.

19. Gaida R.P., Kluchkovsky Yu.B., Tretiak V.I. Lagrangian function and equations of motion of relativistic interacting particle system. In: 9th Intern. conference on general relativity and gravitation. Abstracts of contributed papers for the discussion groups (Jena, July 14-19, 1980), vol. 2, Jena, 1980, p. 164-165.

20. Gaida R.P., Kluchkovsky Yu.B., Tretyak V.I. Lagrangian classical relativistic mechanics of a system of directly interacting particles // Theor. Math. Phys., 1980, vol. 44, No 2, p. 687-697.

21. Gaida R.P., Kluchkovsky Yu.B., Tretyak V.I. Three-dimensional Lagrangian approach to the classical relativistic dynamics of directly interacting particles.- In: Constraint's theory and relativistic dynamics. Eds. G.Longhi, L.Lusanna. Singapore, World Sci. Publ., 1987, p. 210-241.

22. Duviryak A.A., Kluchkovsky Yu.B. Covariant coordinates in the relativistic Hamil- 
tonian mechanics of particle system./Academy of Sciences of the Ukrainian SSR, Institute for Applied Problems of Mechanics \& Mathematics. Preprint No 10-88, Lviv 1988, $21 \mathrm{p}$.

23. Sokolov S.N. Theory of relativistic direct interaction (problems and perspectives)./Preprint IHEP 78-125, Serpukhov 1978, 39 p.

24. Sokolov S.N. Relativistic classical Hamiltonian mechanics in three forms of dynamics. Preprint IHEP 8-78, Serpukhov 1981, 15 p.

25. Currie D.G., Jordan T.F., Sudarshan E.C.G. Relativistic invariance and Hamiltonian theories of interacting particles // Rev. Mod. Phys., 1963, vol. 35, No 2, p. 350-375.

26. Chelkovski S., Nietende J., Suchanek R. No-interaction theorems in relativistic particle dynamics // Acta Phys. Pol., 1980, vol. B11, No 11, p. 809-825.

27. Gaida R.P., Kluchkovsky Yu.B., Tretyak V.I. Forms of relativistic dynamics in classical Lagrangian description of a system of particles // Teor. Mat. Fiz., 1983, vol. 55, No 1, p. 88-105 (in Russian).

28. Gaida R.P., Tretyak V.I. Direct action Lagrangian and Hamiltonian description of a particle system in the distinct forms of relativistic mechanics./Preprint of Institute of Theoretical Physics, ITP-82-87P, Kiev 1982, 38 p. (in Russian).

29. Pauri M., Prosperi G.M. Canonical realizations of the Poincaré group. II. Spacetime description of two particles interacting at a distance, Newtonian-like equations of motion and approximately relativistic Lagrangian formulation // J. Math. Phys., 1976, vol. 17, No 8, p. 1468-1495.

30. Pons J.M. World-lines Poincaré realizations and predictive relativistic mechanics // Ann. Phys. (US), 1983, vol. 148, No 1, p. 192-213.

31. Zhdanov V.I., Opanasiuk Yu.A. Phenomenological formulation of a direct interparticle action in relativistic theory of gravitation // Izv. vuzov. Phys., 1986, No 11, p. 69-73 (in Russian).

32. Opanasiuk Yu.A., Pyragas K.A. Relativistic motion equations and their relation to the equations in metric theories of gravitation // Bulletin of the Vilnius astronomical observatory, 1988, No 81, p. 35-41.

33. Opanasiuk Yu.A. On condition of integration of Currie-Hill equations for many particles systems.-In: Contributed papers of VII Soviet Union Conf. on Modern theoretical and experimental problems of relativity and gravitation. Yerevan, 18-20 October 1988, p. 215-218 (in Russian).

34. Thorne K.S., Will C.M. Theoretical frameworks for testing relativistic gravity. I. Foundations // Astrophys. J., 1971, vol. 163, p. 595-610.

35. Will C.M. Theoretical frameworks for testing relativistic gravity. II. Parametrized post-Newtonian hydrodynamics, and Nordtvedt effect // Astrophys. J., 1971, vol. 163, p. $611-628$.

36. Will C.M. Theoretical frameworks for testing relativistic gravity. III. Conservation laws, Lorentz invariance, and values of the PPN parameters // Astrophys. J., 1971, vol. 169 , p. 125-140.

37. Weinberg S. Gravitation and Cosmology. Wiley, New-York e.a., 1972.

38. Gaida R.P., Tretyak V.I. Single-time form of the Fokker-type relativistic dynamics. I // Acta Phys. Pol., 1980, vol. B11, No 7, p. 509-522.

39. Pyragas K.A., Zhdanov V.I., Alexandrov A.N., Kudrya Yu.N., Pyragas L.E. Qualitative and Analytical Methods in Relativistic Dynamics. Energoizdat, Moscow, 1995 (in Russian). 


\title{
Про одночасові форми релятивістичної динаміки ґравітуючих часток
}

\author{
Ю.А.Опанасюк
}

Національний технічний університет України “Київський

Політехнічний інститут”, 252056 м. Київ, просп. Перемоги, 37

Отримано 4 березня 1998 р.

Подано стислий огляд розвитку Пуанкаре-інваріянтних методів опису механічної системи, що ґрунтуються на сучасній концепції прямої взаємодії. Обговорюється певний підхід до проблеми руху в релятивістичній теорії ґравітації. Підхід суттєво використовує методи предиктивної (з єдиним параметром еволюції) релятивістичної механіки із залученням певних феноменологічних припущень щодо характеру релятивістичних “сил”. Доведено необхідні та достатні умови для побудови в межах обговорюваного підходу наближених (у довільному порядку) розв'язків рівнянь Каррі-Хілла. Для замкнутої системи N ґравітуючих безструктурних точкових часток у пост-ньютонівському наближенні запропоновано узагальнені Пуанкаре-інваріянтні рівняння, які можуть застосовуватися при створенні нового, більш загального, формалізму верифікації релятивістичних теорій тяжіння. Обговорюються взаємозв'язки запропонованих рівнянь та рівнянь, що виникають у лаґранжевому формулюванні релятивістичної динаміки.

Ключові слова: релятивістична механіка, пряма взаємодія, тяжіння

PACS: 04.25. $-\mathrm{g}, 04.80 . \mathrm{Cc}, 11.30 . \mathrm{Cp}$ 
